\title{
Social Isolation and Sleep: Manifestation During COVID-19 Quarantines
}

\author{
June J. Pilcher*, Logan L. Dorsey, Samantha M. Galloway and Dylan N. Erikson \\ Department of Psychology, Clemson University, Clemson, SC, United States
}

Although researchers have investigated the impact of social isolation on well-being, the recent quarantines due to COVID-19 resulted in a social isolation environment that was unique to any examined in the past. Because sleep is one of the endogenous drives that impacts short and long-term health and well-being, it is important to consider how social isolation during the COVID-19 government-mandated quarantines affected sleep and sleep habits. A number of researchers have addressed this question during the last 2 years by examining several concepts related to possible changes in sleep during the quarantines. To best understand these recent results, the current mini review provides a background on the pre-pandemic literature on the effects of social isolation and loneliness with a focus on sleep and then summarizes the recent literature on sleep

OPEN ACCESS

Edited by: Isa Okajima,

Tokyo Kasei University, Japan

Reviewed by:

Yuichi Inoue,

Tokyo Medical University, Japan Hisayoshi Okamura, Kurume University, Japan

*Correspondence: June J. Pilcher jpilche@clemson.edu

Specialty section:

This article was submitted to Personality and Social Psychology, a section of the journal

Frontiers in Psychology

Received: 07 November 2021

Accepted: 20 December 2021

Published: 10 January 2022

Citation:

Pilcher JJ, Dorsey LL, Galloway SM and Erikson DN (2022) Social Isolation and Sleep: Manifestation During COVID-19

Quarantines.

Front. Psychol. 12:810763. doi: 10.3389/fpsyg.2021.810763 and sleep habits. In general, sleep was negatively impacted for many people during the pandemics but not all. One group that seemed to benefit from the pandemic in terms of sleep patterns, were younger people who could more easily adapt their sleep times to match their internal chronobiology. Given the potential broad impact of sleep on health and well-being, better understanding how social isolation impacts sleep is an important consideration for individuals, work organizations, and governments.

Keywords: social isolation, sleep, social relationships, COVID-19, loneliness, review

\section{INTRODUCTION}

Social isolation can arise from many causes. Disruptions to our daily social connections (e.g., job loss) result in people adapting to some level of social isolation. Quarantine procedures used to control virus outbreaks can result in extended social isolation. For example, numerous citywide quarantines were put in place in China and Canada during the 2003 outbreak of severe acute respiratory syndrome (SARS). In the United States, individuals with SARS were placed in individual quarantine. Similarly, many villages in west African countries were quarantined in the 2014 Ebola outbreak (Brooks et al., 2020). Since the new SARS outbreak, COVID-19, in December 2019, many people in numerous countries have been required to quarantine for weeks to months where they were obligated to work at home, home-school children, and drastically decrease social interaction resulting in prolonged periods of enforced social isolation.

The impact of such widespread social isolation is not yet well understood. Some research has, however, focused on the potential impact of meaningful social relationships in humans. The association between positive social relationships and health has been clearly established. Individuals who are well integrated into society typically display lower mortality, lower rates of chronic diseases, and healthier overall behavior (House et al., 1988). Similarly, being socially connected 
positively impacts psychological, emotional, and physical wellbeing (Uchino, 2006). A meta-analytic summary of the literature found that inadequate social relationships is linked with an increase in risk of premature mortality that is similar to wellestablished health-risk factors such as decreased physical activity and increased obesity (Holt-Lunstad et al., 2015).

Good interpersonal relationships could help enable social influence on individual choices and behaviors by providing a structure that encourages individuals to choose better health-related behaviors (Umberson, 1987). For example, social relationships can help one maintain a more consistent sleep routine that can result in better stress management and long-term health benefits. However, the functionality of this type of social structure could be undermined by a quarantine-like scenario such as those implemented to control the spread of COVID-19.

The purpose of this mini review is to assess recent findings pertaining to sleep and sleep habits under COVID-19 quarantine conditions within the broader context of the social isolation research area. To identify the relevant literature, we used Google Scholar and PsycInfo. We also searched the citations within the literature we identified and the "cited by" index in Google Scholar to locate additional relevant studies. Together, these search methods provided a substantial base of literature to inform our assessment of social isolation and sleep.

\section{SOCIAL ISOLATION AND LONELINESS}

Research examining the impact of social isolation is often intermixed with research on perceived loneliness. While the concepts of social isolation and loneliness are sometimes used interchangeably, they can be viewed as two separate scientific constructs. Perceived loneliness is defined by one's inability to fulfill personal social desires (Ernst and Cacioppo, 1999; Cacioppo et al., 2010). Individuals are at greater risk of experiencing perceived loneliness when they have few social networks, conflicts in their current family or intimate relationships, or social connections that are not close enough to be considered a social relationship (Dykstra and Fokkema, 2007; van Tilburg et al., 2015; Gierveld et al., 2018). It is important to note that an individual can be lonely, but not be socially isolated and vice versa (Victor et al., 2000; Cacioppo et al., 2009; Gierveld et al., 2018). While the focus of the current paper is on social isolation, it is important to note that feelings of loneliness are likely part of the broad impact of social isolation resulting from the COVID-19 quarantines.

Although one can be socially isolated and not experience the emotion of loneliness, this is unlikely for most people (Victor et al., 2000; Gierveld et al., 2018). By nature, humans are social entities and when they are isolated from human contact or experience feelings of loneliness, health risks and sleep problems increase (Cacioppo et al., 2000, 2011; Robins et al., 2018; Griffin et al., 2020). In the case of social isolation due to the COVID19 quarantines, it is likely that many individuals maintained some level of family connections but experienced a decrease in other social contact. In addition, many individuals could have maintained some level of social relationships using social media
(Pancani et al., 2021). It seems likely that most individuals had some level of social contact during the COVID-19 quarantines, nonetheless most would have experienced a dramatic decrease in their normal social interactions.

Research suggests that socially isolated individuals are at a greater risk for increased stress and higher mortality and morbidity (House et al., 1982, 1988; Umberson, 1987; Brummett et al., 2001; Cacioppo et al., 2011). Some research equates experiencing social isolation with the same health risks as smoking 15 cigarettes or drinking six alcoholic beverages a day (House et al., 1982; Brummett et al., 2001; Holt-Lunstad et al., 2015; Robins et al., 2018). Social isolation is also highly comorbid with mental disorders like depression, anxiety, and dementia (Ernst and Cacioppo, 1999; Cacioppo et al., 2000; Robins et al., 2018). However, research also indicates that interaction with as little as one other individual can greatly reduce the health risks associated with social isolation (Cacioppo et al., 2000; Brummett et al., 2001), suggesting that the specific characteristics of the social isolation environment can affect the broader impact on individuals.

The exact social isolation conditions resulting from COVID19 quarantines varied across different individuals and countries. Some individuals could have been isolated with their families while other individuals could have been living alone but using social media to maintain some social contact. Similarly, some countries repeated quarantines over time while other countries had longer, sustained quarantines in place. As such, the social isolation with the resultant feelings of loneliness experienced during COVID-19 quarantines is unique and presents an environment unlike the types of social isolation seen in previous studies.

\section{SLEEP AND SLEEP HABITS}

Pre-COVID-19 pandemic studies have shown that social isolation negatively impacts sleep (Cacioppo et al., 2002; Kurina et al., 2011). Furthermore, a recent review of the prepandemic literature on social relationships and sleep concluded that increased quantity and quality of mutually supportive relationships are positively related to sleep (Gordon et al., 2021). It is also well established that sleep and sleep habits can be negatively impacted by psychosocial stress (Åkerstedt, 2006) as occurs during prolonged social isolation as seen in the COVID-19 quarantines (Yuksel et al., 2021).

Sleep is a health behavior that is highly predicated on a consistent routine and thus, could be compromised as a result of the changes in lifestyle during a quarantine. The implication of this relationship could be significant, given the evidence in the scientific community that links poor sleep to poor health outcomes. Sleep disturbances, unrelated to an underlying medical condition, are associated with increased cardiovascular disease, cancer, and obesity as well as to allcause mortality (Cappuccio et al., 2010). Sleep disturbances also negatively impact psychological functioning, immune response, and mood regulation (Medic et al., 2017). In addition, sleep habits are a better predictor of mental health and well-being than 
other health-related behaviors such as physical activity and diet (Wickham et al., 2020).

Sleep loss can also have more acute effects on health. For example, a single night of simulated shift work results in higher blood pressure among young adults at risk for hypertension (McCubbin et al., 2010) and alters respiratory sinus arrhythmia when completing complex tasks (Walker et al., 2009). As such, maintaining good sleep habits and adequate sleep would be an important component of good mental and physical health both in the short-term, such as during a quarantine, but also lifelong.

It is important to note that sleep quality is at least as important to good health as sleep quantity. Sleep quality is often the better predictor of mental health and well-being (Wickham et al., 2020) and is more closely associated with health, affect, and life satisfaction than sleep quantity (Pilcher et al., 1997) particularly in persons sleeping between 6 and $8 \mathrm{~h}$ a night. In addition, both social isolation and perceived loneliness negatively impact sleep quality (Matthews et al., 2017; Cho et al., 2019). It has also been shown in older individuals that social isolation predicts poor sleep quality 6 years in the future (Yu et al., 2018). Therefore, understanding the interaction between sleep and social relationships is important in understanding how humans' social nature impact our health and well-being (Cacioppo et al., 2011; Robins et al., 2018).

\section{SOCIAL ISOLATION AND SLEEP DURING COVID-19}

Understanding the impact of government-mandated quarantines that extended across many countries and millions of people on sleep is crucial due to the many links between sleep, health, and well-being. Because modern technology has enabled forms of communication that make total isolation less likely, the COVID19 quarantines may not have resulted in total social isolation for many people, however, the quarantines would have caused unique challenges leading to changes in daily life for most people. Furthermore, recent research indicates that people experienced increased feelings of depression and anxiety during the COVID19 quarantine (Peterson et al., 2021) which could negatively impact sleep and sleep habits for many individuals.

\section{Meta-Analyses and Large Multi-Country Studies}

A number of review articles and multi-country studies have focused specifically on sleep and sleep patterns during the COVID-19 quarantine. One meta-analysis examining sleep habits across 13 countries found that $35.7 \%$ of the participants experienced sleep disturbances (Jahrami et al., 2021). Another meta-analysis examined gender differences in sleep problems across 19 countries and found that $24 \%$ of female participants and $27 \%$ of male participants experienced sleep problems during the quarantines (Alimoradi et al., 2021).

Several multi-country studies included specific information about sleep habits that was not easily captured in the meta-analyses. One multi-country study found that over 50\% of the participants delayed their sleep and wake times with more than a third of the participants reporting increased sleep disturbances (Yuksel et al., 2021). In addition, they concluded that poorer sleep was associated with increased depression and anxiety symptoms. Another multi-country study reported that equal numbers of participants indicated no change in sleep as worsening sleep (Mandelkorn et al., 2021). In this study, the people most likely to report increased sleep disturbances were women, people between the ages of 31 and 45, and people who were less physically active. One multi-country study found that sleep was seen as less restful and of lower quality during the quarantine than prior to the quarantine and that decreases in sleep quality were negatively correlated with perceived social isolation, depression, and anxiety (Salah et al., 2021). Finally, a multi-country study that included many high school students found that most changes in sleep patterns occurred within the first 2 weeks of the quarantine (AMHSI Research Team et al., 2020). More specifically, they reported that the differences between sleep duration on weekdays versus weekends disappeared with total sleep time increasing by about one hour during the quarantine particularly for the adolescents.

These large-scale studies provide a good foundation for understanding the impact of social isolation during the COVID19 quarantine on sleep and sleep habits. These studies reported that people often indicate worse sleep quality or sleep problems during the quarantine. However, there were also some people, particularly older adolescents, who increased their sleep time. In addition, there were differing reports on the prevalence of sleep problems in females and males.

\section{Primary Studies}

Other studies, though individually less encompassing than the reviews and multi-country studies, provide important information about the impact of social isolation during the COVID-19 quarantines on sleep. In this section, we focus on primary studies that were published more recently and were not captured in the meta-analyses described above to provide a broad picture of what the current literature is showing.

Many sleep scientists used sleep quality or similar constructs to document changes in sleep during the COVID-19 quarantines. Many studies across numerous countries reported a worsening of sleep quality, increasing sleep problems, or increasing problems with insomnia during quarantine conditions (Blume et al., 2020; Peretti-Watel et al., 2020; Pinto et al., 2020; Voitsidis et al., 2020; AlRasheed et al., 2021; Cellini et al., 2021; Conte et al., 2021; Hisler and Twenge, 2021; Hyun et al., 2021; Marelli et al., 2021; Martínez-de-Quel et al., 2021; Robillard et al., 2021). However, some studies found that sleep quality did not worsen across all participants (Benham, 2020; Gao and Scullin, 2020; Kocevska et al., 2020; Tahara et al., 2021). Of these studies, a common trend was that younger people or college students reported less sleep problems during the quarantine (Benham, 2020; Tahara et al., 2021).

In addition to quality-type measures, many researchers examined changes in sleep patterns during the COVID-19 quarantines. Delays in sleep timing which frequently included a concomitant increase in sleep quantity were commonly found across many countries (Benham, 2020; Blume et al., 2020; 
Sinha et al., 2020; Wright et al., 2020; Cellini et al., 2021; Conte et al., 2021; Robillard et al., 2021; Tahara et al., 2021). The delays in sleep timing included later bedtimes, mid-sleep times, and wake-up times often resulting in less social jetlag (where sleep times differ between workdays and non-workdays). One study found that the number of college students reporting seven or more hours of sleep a night increased from 84 to $92 \%$ for weekdays during the quarantine (Wright et al., 2020). Not surprisingly, there was variability in the responses from participants about time in bed during the pandemic. One study reported that although average wake time was delayed, some participants reported a decrease in total sleep time (Robillard et al., 2021) while a different study focusing on adults over 60 years old found that about $27 \%$ of participants reported more sleep than usual while about $15 \%$ reported less sleep than usual during the pandemic (Emerson, 2020). Another study found that average time asleep did not change during the pandemic, however, there was greater prevalence of both shorter and longer sleep than the recommended $7-8 \mathrm{~h}$ a night (Hisler and Twenge, 2021).

Another area of sleep-related research during the COVID-19 quarantines concerned the potential impact of variables related to the characteristics of the individual participants. This included differences between females and males, personality types, stress, and mood variables. In general, women were more likely to experience worse sleep quality and worse insomnia than men across many countries (Pinto et al., 2020; Voitsidis et al., 2020; Cellini et al., 2021; Marelli et al., 2021; Robillard et al., 2021). In addition, women were more likely to experience increased feelings of distress and loneliness during the quarantines (LosadaBaltar et al., 2021). In contrast, one study found that although women reported more anxiety than men, their sleep quality did not differ (Bigalke et al., 2020).

Lower quality sleep and insomnia were correlated with depressive symptoms and feelings of loneliness (Voitsidis et al., 2020; Hyun et al., 2021), feelings of anxiety (Xiao et al., 2020), a more negative mood (Ingram et al., 2020; Cellini et al., 2021), and negative affect and worry (Kocevska et al., 2020). Furthermore, some studies divided the participants into groups depending on sleep-related variables or personality-type variables. One study divided their participants into three groups based on sleep quantity (reduced or extended) and delayed sleep times (Robillard et al., 2021). They concluded that those participants experiencing a reduction in sleep quantity or delayed sleep times also experienced more stress, anxiety, and depression during the quarantines. Another study divided their participants into three groups based on adaptive-type personality profiles (Ahmed et al., 2021). They found that persons with highly adaptive personalities exhibited less perceived stress and better sleep quality while persons with a maladaptive personality profile experienced the highest perceived stress and poorer sleep quality.

\section{CONCLUSION AND IMPLICATIONS}

The drastic increase in levels of social isolation during the COVID-19 quarantines was accompanied by changes in sleep.
Sleep quality suffered, particularly in women. Interestingly, the timing of sleep changed with some people, especially younger adults, shifting their bed and wake up time to later in the day while often increasing the time asleep. This suggests that many people curtail their sleep to meet the time demands of their workplace or education settings and that the quarantines provided flexibility, thus allowing some individuals to better match their sleep to their chronobiological drives. It is also important to note that not everyone experienced an increase in sleep quantity during the quarantines. Some people, particularly those who reported feelings of depression, anxiety, loneliness, or stress, experienced less sleep as well as worse sleep quality.

As noted earlier, social isolation could include feelings of loneliness for some people. Unfortunately, this manuscript could not address this issue since few studies on sleep during the COVID-19 quarantines examined loneliness. Those studies that did suggested that feelings of loneliness were related to increased incidences of disturbed sleep. However, future research is needed to better document the potential impact of loneliness on sleep under social isolation conditions.

As would be expected, sleep and sleep habits varied across individuals during the COVID-19 quarantines. Some of the differences in sleep disruptions were related to individual characteristics such as differences between females and males or a different adaptation in sleep behaviors for younger adults than other adults. There are other variables that could have moderated the impact of social isolation on sleep such as social support. Studies indicate that during the COVID-19 quarantines perceived social support served as a buffer for the negative impact of the quarantines on sleep, stress, anxiety, and depression (Grey et al., 2020; Li et al., 2020; Xiao et al., 2020). A lack of social support can also cause a decline in self-control ability (Pilcher and Bryant, 2016), which in turn could lead to poorer decisions regarding sleep habits (Pilcher et al., 2015). This sequence of events could contribute to worse sleep under social isolation conditions for individuals who experience less social support. Another potential moderating variable is exercise which has been shown to be related to sleep quality and quantity (Kovacevic et al., 2018; Pilcher et al., 2021). Unfortunately, exercise was not a common measure for the quarantine-based studies on sleep and, thus, could not be assessed here. Other potential moderating variables could be family and work obligations, preexisting medical conditions, or neurological conditions. Unfortunately, these types of constructs were not commonly measured in the quarantine-based studies on sleep. Additional research is needed to document the interactions between potential moderating variables and the impact of social isolation on sleep.

The literature reviewed here suggests that a complex, multidimensional relationship exists that connects social integration and sleep. In general, social isolation during the quarantines negatively impacted sleep for many individuals which could then have short- and long-term consequences on health. Given that interventions in one health area could positively impact other health areas (Rajkumar, 2020), it is important to consider the potential impact of developing interventions that address the range of sleep problems that can emerge 
during social isolation conditions. This can include interventions from governments, workplaces, and health-care professionals that incorporate educational efforts, treatment of insomnia, chronobiological therapies, and cognitive-behavioral therapies that can help individuals more readily adapt to social isolation.

The current mini review supports the importance of understanding the broader impact of social isolation on sleep as well as the need for additional research. It is important to note that social isolation could occur in a variety of settings other than a pandemic, including isolation because of personal decisions to withdraw from others, disruption in a normal social group such as when losing a job, mental disorders, retirement, aging, and when working primarily from home. Government, workplace, and health-care interventions are needed that are broadly accessible by the public to assist individuals in better

\section{REFERENCES}

Ahmed, O. Hossain, K. N., Siddique, R. F. and Jobe, M. C. (2021). COVID-19 fear, stress, sleep quality and coping activities during lockdown, and personality traits: a person-centered approach analysis. Pers. Individ. Dif. 178:110873. doi: 10.1016/j.paid.2021.110873

Åkerstedt, T. (2006). Psychosocial stress and impaired sleep. Scand J. Work Environ. Health 32, 493-501. doi: 10.5271/sjweh.1054

Alimoradi, Z., Gozal, D., Tsang, H. W. H., Lin, C., Brostrom, A., Ohayon, M. M., et al. (2021). Gender-specific estimates of sleep problems during the COVID19 pandemic: systematic review and meta-analysis. J. Sleep Res. 00:e13432. doi: $10.1111 /$ jsr.13432

AlRasheed, M. M., Alkadir, A. M., Bin Shuqiran, K. I., Al-Aqeel, S., Jahrami, H. A., and BaHammam, A. S. (2021). The impact of quarantine on sleep quality and psychological distress during the COVID-19 pandemic. Nat. Sci. Sleep 13, 1037-1048. doi: 10.2147/NSS.S313373

AMHSI Research Team, Milken Research Team, Roitblat, Y., Burger, J., Leit, A., Nehuliaieva, L., et al. (2020). Stay-at-home circumstances do not produce sleep disorders: an international survey during the COVID-19 pandemic. J. Psychosom. Res. 139:110282. doi: 10.1016/j.jpsychores.2020.110282

Benham, G. (2020). Stress and sleep in college students prior to and during the COVID-19 pandemic. Stress Health 27, 504-515. doi: 10.1002/smi.3016

Bigalke, J. A., Greenlund, I. M., and Carter, J. R. (2020). Sex differences in selfreport anxiety and sleep quality during COVID-19 stay-at-home orders. Bio Sex Differ 11:56. doi: 10.1186/s13293-020-00333-4

Blume, C., Schmidt, M. H., and Cajochen, C. (2020). Effects of the COVID-19 lockdown on human sleep and rest-activity rhythms. Curr. Biol. 30, R795-R797. doi: $10.1016 /$ j.cub.2020.06.021

Brooks, S. K., Webster, R. K., Smith, L. E., Woodland, L., Wessely, S., Greenberg, N., et al. (2020). The psychological impact of quarantine and how to reduce it: rapid review of the evidence. Lancet 395, 912-920. doi: 10.1016/S0140-6736(20) 30460-8

Brummett, B. H., Barefoot, J. C., Siegler, I. C., Clapp-Channing, N. E., Lytle, B. L., Bosworth, H. B., et al. (2001). Characteristics of socially isolated patients with coronary artery disease who are at elevated risk for mortality. Psychosom. Med. 63, 267-272. doi: 10.1097/00006842-200103000-0 0010

Cacioppo, J. T., Ernst, J. M., Burleson, M. H., McClintock, M. K., Malarkey, W. B., Hawkley, L. C., et al. (2000). Lonely traits and concomitant physiological processes: The MacArthur social neuroscience studies. Int. J. Psychophysiol. 35, 143-154. doi: 10.1016/S0167-8760(99)00049-5

Cacioppo, J. T., Fowler, J. H., and Christakis, N. A. (2009). Alone in the crowd: The structure and spread of loneliness in a large social network. J. Pers. Soc. Psychol. 97, 977-991. doi: 10.1037/a0016076

Cacioppo, J. T., Hawkley, L. C., Berntson, G. G., Ernst, J. M., Gibbs, A. C., Stickgold, R., et al. (2002). Do lonely days invade the nights? potential social modulation of sleep efficiency. Psychol. Sci. 13, 384-387. doi: 10.1111/1467-9280.00469 managing their health behaviors including their sleep habits when experiencing social isolation.

\section{AUTHOR CONTRIBUTIONS}

JP completed the final drafts of the manuscript. All authors were part of the conceptualization of the manuscript and worked on earlier drafts.

\section{FUNDING}

Funds from the Creative Inquiry and Undergraduate Research Program at Clemson University for open access publication fees.

Cacioppo, J. T., Hawkley, L. C., Norman, G. J., and Berntson, G. G. (2011). Social isolation. Ann. NY Acad. Sci. 1231, 17-22. doi: 10.1111/j.1749-6632.2011.06 028.x

Cacioppo, J. T., Hawkley, L. C., and Thisted, R. A. (2010). Perceived social isolation makes me sad: Five year cross-lagged analyses of loneliness and depressive symptomatology in the Chicago Health. Aging, and Social Relations study. Psychol. Aging 25, 453-463. doi: 10.1037/a001 7216

Cappuccio, F. P., D’Elia, L., Strazzullo, P., and Miller, M. A. (2010). Sleep duration and all-cause mortality: a systematic review and meta-analysis of prospective studies. Sleep 33, 585-592.

Cellini, N., Conte, F., De Rosa, O., Giganti, F., Malloggi, S., Reyt, M., et al. (2021). Changes in sleep timing and subjective sleep quality during the COVID-19 lockdown in Italy and Belgium: Age, gender and working status as modulating factors. Sleep Med. 77, 112-119. doi: 10.1016/j.sleep.2020.11.027

Cho, J. H., Olmstead, R., Choi, H., Carrillo, C., Seeman, T. E., and Irwin, M. R. (2019). Associations of objective versus subjective social isolation with sleep disturbance, depression, and fatigue in community-dwelling older adults. Aging Ment. Health 23, 1130-1138. doi: 10.1080/13607863.2018.1481928

Conte, F., Cellini, N., De Rosa, O., Rescott, M. L., Malloggi, S., Giganti, F., et al. (2021). Dissociated profiles of sleep timing and sleep quality changes across the first and second wave of the COVID-19 pandemic. J. Psychiatr. Res. 143, 222-229. doi: 10.1016/j.jpsychires.2021.09.025

Dykstra, P. A., and Fokkema, T. (2007). Social and emotional loneliness among divorced and married men and women: comparing the deficit and cognitive perspectives. Basic Appl. Soc. Psych. 29, 1-12. doi: 10.1080/01973530701330843

Emerson, K. G. (2020). Coping with being cooped up: Social distancing during COVID-19 among 60+ in the United States. Pan Am. J. Public Health 44:81. doi: $10.26633 /$ RPSP.2020.81

Ernst, J. M., and Cacioppo, J. T. (1999). Lonely hearts: psychological perspectives on loneliness. Appl. Prev. Psychol. 8, 1-22. doi: 10.1016/S0962-1849(99)80008-0

Gao, C., and Scullin, M. K. (2020). Sleep health early in the coronavirus disease 2019 (COVID-19) outbreak in the United States: Integrating longitudinal, cross-sectional, and retrospective recall data. Sleep Med. 73, 1-10. doi: 10.1016/ j.sleep.2020.06.032

Gierveld, J. D. J., Tilburg, T. G., and Dykstra, P. A. (2018). "New ways of theorizing and conducting research in the field of loneliness and social isolation," in The Cambridge Handbook of Personal Relationships, eds A. L. Vangelisti and D. Perlman (Cambridge, MA: Cambridge University Press), 391-404. doi: 10. 1017/9781316417867.031

Gordon, A. M., Carrillo, B., and Barnes, C. M. (2021). Sleep and social relationships in healthy populations: a systematic review. Sleep Med. Rev. 57:101428. doi: 10.1016/j.smrv.2021.101428

Grey, I., Arora, T., Thomas, J., Saneh, A., Tohme, P., and Abi-Habib, R. (2020). The role of perceived social support on depression and sleep during the COVID19 pandemic. Psychiatry Res. 293:113452. doi: 10.1016/j.psychres.2020.11 3452 
Griffin, S. C., Williams, A. B., Ravyts, S. G., Mladen, S. N., and Rybarczyk, B. D. (2020). Loneliness and sleep: A systematic review and meta-analysis. Health Psychol. Open 7:2055102920913235. doi: 10.1177/2055102920913235

Hisler, G. C., and Twenge, J. M. (2021). Sleep characteristics of U.S. adults before and during the COVID-19 pandemic. Soc. Sci. Med. 276:113849. doi: 10.1016/j. socscimed.2021.113849

Holt-Lunstad, J., Smith, T. B., Baker, M., Harris, T., and Stephenson, D. (2015). Loneliness and social isolation as risk factors for mortality: a meta-analytic review. Perspect. Psychol. Sci. 10, 227-237. doi: 10.1177/1745691614568352

House, J. S., Landis, K. R., and Umberson, D. (1988). Social relationships and health. Science 241, 540-545. doi: 10.1136/sextrans-2018-053935

House, J. S., Robbins, C., and Metzner, H. L. (1982). The association of social relationships and activities with mortality: prospective evidence from the Tecumseh Community Health study. Am. J. Epidemiol. 116:123. doi: 10.1093/ oxfordjournals.aje.a113387

Hyun, S., Hahm, H. C., Wong, G. T. F., Zhang, E., and Liu, C. H. (2021). Psychological correlates of poor sleep quality among U.S. young adults during the COVID-19 pandemic. Sleep Med. 78, 51-56. doi: 10.1016/j.sleep.2020.12. 009

Ingram, J., Maciejewski, G., and Hand, C. J. (2020). Changes in diet, sleep, and physical activity are associated with differences in negative mood during COVID-19 lockdown. Front. Psychol. 11:588604. doi: 10.3389/fpsyg.2020. 588604

Jahrami, H., BaHammam, A. S., Bragazzi, N. L., Saif, Z., Faris, M., and Vitiello, M. V. (2021). Sleep problems during the COVID-19 pandemic by population: a systematic review and meta-analysis. J. Clin. Sleep Med. 17, 299-313. doi: $10.5664 /$ jcsm. 8930

Kocevska, D., Blanken, T. F., Van Someren, E. J. W., and Rösler, L. (2020). Sleep quality during the COVID-19 pandemic: not one size fits all. Sleep Med. 76, 86-88. doi: 10.1016/j.sleep.2020.09.029

Kovacevic, A., Mavros, Y., Heisz, J. J., and Fiatarone Singh, M. A. (2018). The effect of resistance exercise on sleep: a systematic review of randomized controlled trials. Sleep Med. Rev. 39, 52-68. doi: 10.1016/j.smrv.2017.07.002

Kurina, L. M., Knutson, K. L., Hawkley, L. C., Cacioppo, J. T., Lauderdale, D. S., and Ober, C. (2011). Loneliness is associated with sleep fragmentation in a communal society. Sleep 34, 1519-1526. doi: 10.5665/sleep.1390

Li, X., Wu, H., Meng, F., Li, L., Wang, Y., and Zhou, M. (2020). Relations of COVID-19-related stressors and social support with Chinese college students' psychological response during the COVID-19 pandemic. Front. Psychiatry 11:551315. doi: 10.3389/fpsyt.2020.551315

Losada-Baltar, A., Jiménez-Gonzalo, M. A., Gallego-Alberto, L., del Sequeros Pedroso-Chaparro, M., Fernandes-Pires, J., and Márquez-González, M. (2021). We are staying at home." Associations of self-perceptions of aging, personal and family resources, and loneliness with psychological distress during the lockdown period of COVID-19. J. Gerontol. B. Psychol. Sci. Soc. Sci. 76, e10-e16. doi: 10.1093/geronb/gbaa048

Mandelkorn, U., Genzer, S., Choshen-Hillel, S., Reiter, J., Cruz, M. M., Hochner, H., et al. (2021). Escalation of sleep disturbances amid the COVID-19 pandemic: a cross sectional international study. J. Clin. Sleep Med. 17, 45-53. doi: 10.5664/ jcsm. 8800

Marelli, S., Castelnuovo, A., Somma, A., Castronovo, V., Mombelli, S., Bottoni, D., et al. (2021). Impact of COVID-19 lockdown on sleep quality in university students and administration staff. J. Neurol. 268, 8-15. doi: 10.1007/s00415020-10056-6

Martínez-de-Quel, O., Suarez-Iglesias, D., Lopez-Flores, M., and Perez, C. A. (2021). Physical activity, dietary habits and sleep quality before and during COVID-19 lockdown: A longitudinal study. Appetite 158:105019. doi: 10.1016/ j.appet.2020.105019

Matthews, T., Danese, A., Gregory, A. M., Caspi, A., Moffitt, T. E., and Arseneault, L. (2017). Sleeping with one eye open: Loneliness and sleep quality in young adults. Psychol. Med. 47, 2177-2186. doi: 10.1017/S003329171700 0629

McCubbin, J. A., Pilcher, J. J., and Moore, D. D. (2010). Blood pressure increases during a simulated night shift in persons at risk for hypertension. Int. J. Behav. Med. 17, 314-320. doi: 10.1007/s12529-010-9117-6

Medic, G., Wille, M., and Hemels, M. E. (2017). Short- and long-term health consequences of sleep disruption. Nat. Sci. Sleep 9, 151-161. doi: 10.2147/NSS. S134864
Pancani, L., Marinucci, M., Aureli, N., and Riva, P. (2021). Forced social isolation and mental health: a study on 1006 Italians under COVID-19 lockdown. Front. Psychol. 12:663799. doi: 10.3389/fpsyg.2021.663799

Peretti-Watel, P., Alleaume, C., Léger, D., Beck, F., Verger, P., and The Coconel Group. (2020). Anxiety, depression and sleep problems: A second wave of COVID-19. Gen. Psychiatr. 33:e100299. doi: 10.1136/gpsych-2020-10 0299

Peterson, J. A., Chesbro, G., Larson, R., Larson, D., and Black, C. D. (2021). Shortterm analysis (8 weeks) of social distancing and isolation on mental health and physical activity behavior during COVID-19. Front. Psychol. 12:652086. doi: 10.3389/fpsyg.2021.652086

Pilcher, J. J., and Bryant, S. A. (2016). Implications of social support as a self-control resource. Front. Behav. Neurosci. 10:228. doi: 10.3389/fnbeh.2016.00228

Pilcher, J. J., Erikson, D. N., and Yochum, C. A. (2021). Fighting the freshman fifteen: Sleep, exercise, and BMI in college students. Am. J. Health Promot. 35, 580-583. doi: 10.1177/0890117120969065

Pilcher, J. J., Ginter, D. R., and Sadowsky, B. (1997). Sleep quality versus sleep quantity: Relationships between sleep and measures of health, well-being and sleepiness in college students. J. Psychosom. Res. 42, 583-596. doi: 10.1016/ S0022-3999(97)00004-4

Pilcher, J. J., Morris, D. M., Donnelly, J., and Feigl, H. B. (2015). Interactions between sleep habits and self-control. Front. Hum. Neurosci. 9:284. doi: 10.3389/ fnhum.2015.00284

Pinto, J., van Zeller, M., Amorim, P., Pimentel, A., Dantas, P., Eusébio, E., et al. (2020). Sleep quality in times of Covid-19 pandemic. Sleep Med. 74, 81-85. doi: 10.1016/j.sleep.2020.07.012

Rajkumar, R. P. (2020). COVID-19 and mental health: A review of the existing literature. Asian J. Psychiat. 52:102066.

Robillard, R., Dion, K., Pennestri, M., Solomonova, E., Lee, E., Saad, M., et al. (2021). Profiles of sleep changes during the COVID-19 pandemic: demographic, behavioural and psychological factors. J. Sleep Res. 30:13231. doi: 10.1111 /jsr.13231

Robins, L. M., Robins, L. M., Hill, K. D., Finch, C. F., Clemson, L., and Haines, T. (2018). The association between physical activity and social isolation in community-dwelling older adults. Aging Ment. Health 22, 175-182. doi: 10. 1080/13607863.2016.1242116

Salah, A. B., DeAngelis, B. N., and Al'Absi, M. (2021). Resilience and the role of depressed and anxious mood in the relationship between perceived social isolation and perceived sleep quality during the COVID19 Pandemic. Int. J. Behav. Med. 28, 277-285. doi: 10.1007/s12529-020-09 945-x

Sinha, M., Pande, B., and Sinha, R. (2020). Association of mid sleep time and social jetlag with psychosocial behavior of Indian population during COVID-19 lockdown. J. Public Health Res. 9:1870. doi: 10.4081/jphr.2020.1870

Tahara, Y., Shinto, T., Inoue, K., Roshanmehr, F., Ito, A., Michie, M., et al. (2021). Changes in sleep phase and body weight of mobile health App users during COVID-19 mild lockdown in Japan. J. Obes 45, 2277-2280. doi: 10.1038/ s41366-021-00890-7

Uchino, B. N. (2006). Social support and health: a review of physiological processes potentially underlying links to disease outcomes. J. Behav. Med. 29, 377-387. doi: 10.1007/s10865-006-9056-5

Umberson, D. (1987). Family status and health behaviors: social control as a dimension of social integration. J. Health Soc. Behav. 28, 306-319.

van Tilburg, T. G., Aartsen, M. J., and van der Pas, S. (2015). Loneliness after divorce: a cohort comparison among dutch young-old adults. Eur. Sociol. Rev. 31, 243-252. doi: 10.1093/esr/jcu086

Victor, C., Scambler, S., Bond, J., and Bowling, A. (2000). Being alone in later life: Loneliness, social isolation and living alone. Rev. Clin. Gerontol. 10, 407-417. doi: 10.1017/S0959259800104101

Voitsidis, P., Gliatas, I., Bairachtari, V., Papadopoulou, K., Papageorgiou, G., Parlapani, E., et al. (2020). Insomnia during the COVID-19 pandemic in a Greek population. Psychiatry Res. 289:113076.

Walker, A. D., Muth, E. R., Odle-Dusseau, H. N., Moore, D., and Pilcher, J. J. (2009). The effects of 28 hours of sleep deprivation on respiratory sinus arrhythmia during tasks with low and high controlled attention demands. Psychophysiology 46, 217-224. doi: 10.1111/j.1469-8986.2008.00718.x

Wickham, S., Amarasekara, N. A., Bartonicek, A., and Conner, T. S. (2020). The big three health behaviors and mental health and well-being among young 
adults: A cross-sectional investigation of sleep, exercise, and diet. Front. Psychol. 11:579205. doi: 10.3389/fpsyg.2020.579205

Wright, K. P., Linton, S. K., Withrow, D., Casiraghi, L., Lanza, S. M., delaIglesia, H., et al. (2020). Sleep in university students prior to and during COVID-19 Stay-at-Home orders. Curr. Biol. 30, R797-R798. doi: 10.1016/j.cub.2020.06. 022

Xiao, H., Zhang, Y., Kong, D., Li, S., and Yang, N. (2020). Social capital and sleep quality in individuals who self-isolated for 14 days during the coronavirus disease 2019 (COVID-19) outbreak in January 2020 in China. Med. Sci. Monit. 26, e923921-e923921. doi: 10.12659/MSM.923921

Yu, B., Steptoe, A., Niu, K., Ku, P., and Chen, L. (2018). Prospective associations of social isolation and loneliness with poor sleep quality in older adults. Qual. Life Res. 27, 683-691. doi: 10.1007/s11136-017-1752-9

Yuksel, D., McKee, G. B., Perrin, P. B., Alzueta, E., Caffarra, S., Ramos-Usuga, D., et al. (2021). Sleeping when the world locks down: correlates of sleep health during the COVID-19 pandemic across 59 countries. Sleep Health 7, 137-142. doi: $10.1016 /$ j.sleh.2020.12.008
Conflict of Interest: The authors declare that the research was conducted in the absence of any commercial or financial relationships that could be construed as a potential conflict of interest.

Publisher's Note: All claims expressed in this article are solely those of the authors and do not necessarily represent those of their affiliated organizations, or those of the publisher, the editors and the reviewers. Any product that may be evaluated in this article, or claim that may be made by its manufacturer, is not guaranteed or endorsed by the publisher.

Copyright (® 2022 Pilcher, Dorsey, Galloway and Erikson. This is an open-access article distributed under the terms of the Creative Commons Attribution License (CC BY). The use, distribution or reproduction in other forums is permitted, provided the original author(s) and the copyright owner(s) are credited and that the original publication in this journal is cited, in accordance with accepted academic practice. No use, distribution or reproduction is permitted which does not comply with these terms. 